\title{
Valorización de residuos de sílice para la obtención de vidrio soluble mediante análisis térmico diferencial
}

\author{
L. Agudo*, N. Cornejo**, N. Ayala** y F. García-Carcedo**
}

\begin{abstract}
Resumen En la industria del silicio metálico se genera como residuo microsílice, de granulometría muy fina, con alto contenido en sílice $(>92 \%)$. En el presente trabajo se estudia la posibilidad de sustituir, completa o parcialmente, las arenas comerciales por microsílice para la fabricación de vidrio soluble por fusión. Este estudio se lleva a cabo mediante el análisis termogravimétrico y térmico diferencial de las materias primas y mezclas de estas. El balance energético del sistema indica que al utilizar la microsílice requiere el aporte de menor energía externa. La presencia de impurezas en la microsílice puede dar lugar a la formación de compuestos insolubles en el vidrio de silicato. Se concluye que la microsílice puede ser utilizada parcialmente en la elaboración de silicato sódico.
\end{abstract}

Palabras clave Vidrio soluble. Silicato sódico. Microsílice. Valorización.

\section{Valorization of silica fumes to obtainment water glass by thermal analysis}

\begin{abstract}
In the industry of the silicon it is generated as residual silica, of very fine size, with high content in $\mathrm{SiO}_{2}(>92 \%)$. In this work, the possibility of substituting, complete or partially, the residual silica for commercial sands is studied. At the moment, sands are used in the elaboration of soluble glasses by melting. This study is carried out by thermogravimetric and differential thermal analysis of the raw materials and mixtures of them. The energy balance of the system indicates that it is required lower energy when using the residual silica. The presence of impurities in the residual silica can give place to the formation of compound insoluble in the water glass. It is concluded that the residual silica can be used partially in the making of sodium silicate glass.
\end{abstract}

Keywords Water glass. Sodium silicate. Silica. Valorization.

\section{INTRODUCCIÓN}

En la actualidad, los vidrios solubles son muy utilizados en diversas aplicaciones, ya sea a nivel industrial o comercial, tales como la elaboración de adhesivos, recubrimientos anticorrosivos, tratamiento de minerales por flotación, en la industria del papel, en la metalúrgica y refractarios, etc. ${ }^{[1 \text { y } 2]}$. Para producir estos vidrios solubles, como el silicato de sodio de módulo mayor de 3, se utilizan materias primas que aporten sílice y sodio. El principal proceso de elaboración de estos silicatos consiste en la fusión de la mezcla de arena y carbonato sódico, llevado a cabo a altas temperaturas, entre 1.200 y $1.400^{\circ} \mathrm{C}^{[2 \text { y 3]. }}$.
En la industria de la fabricación de silicio metálico se obtiene un residuo denominado "microsílice", un material cuyo contenido en sílice sobrepasa el $90 \%$ y su granulometría está mayoritariamente entre 15 y 30 micrometros. Este residuo representa el $16 \%$ del material generado en esta industria y, actualmente, tiene muy pocas aplicaciones ${ }^{[4]}$.

En el presente trabajo se presentan, los resultados de un estudio llevado a cabo para determinar si esta microsílice, gracias a su alto contenido en $\mathrm{SiO}_{2}$, puede ser utilizada como materia prima para la elaboración de vidrio soluble de alto módulo por el método convencional de fusión. El estudio se ha llevado a cabo en un equipo de análisis térmico.

(*) Universidad Central de Venezuela, Facultad de Ingeniería. Los Chaguaramos, Caracas 1042-A, Venezuela. le cyberleo@yahoo.com

(**) Centro Nacional de Investigaciones Metalúrgicas (CENIM-CSIC), Avda. Gregorio del Amo 8, 28040 Madrid. niloc@cenim.csic.es 
La aplicación de arenas en metalurgia es bien conocida y continúan desarrollándose nuevas aplicaciones $^{[5]}$.

\section{PROCEDIMIENTO EXPERIMENTAL}

La composición química de la materia prima utilizada se observa en la tabla I. Se ha utilizado un equipo de análisis termogravimétrico (TG) y térmico diferencial (ATD) de marca Netzsch. Todos los experimentos se realizaron en una atmósfera oxidante con un flujo de oxígeno de $100 \mathrm{ml} / \mathrm{min}$. Los pesos de las muestras fueron de, aproximadamente, $35 \mathrm{mg}$. Mediante esta técnica se ha estudiado y comparado el proceso de descomposición del carbonato de sodio al interactuar con las distintas fuentes de sílice para dar lugar a la formación de vidrio soluble de silicato de sodio. Se ha utilizado polvo de alúmina como material de referencia y crisoles de alúmina de $0,3 \mathrm{ml}$ de volumen.

En todos los casos las temperaturas máximas alcanzadas en la termobalanza fueron de $1.300^{\circ} \mathrm{C}$ y la velocidad de calentamiento fue de $10^{\circ} \mathrm{C} / \mathrm{min}$. La microsílice fue tratada previamente y analizada por difracción de rayos X (DRX) para la determinación de las fases constituyentes. Para evaluar la formación del vidrio soluble se realizaron ensayos de fusión en un horno vertical, a $1.380^{\circ} \mathrm{C}$ durante $3 \mathrm{~h}$ utilizando un crisol de mullita. Después de vertida la masa fundida se realiza la disolución del vidrio en un autoclave a 8 bares.

En la tabla I, también se da la composición química del carbonato sódico, producto base para la

Tabla I. Análisis químico de las materias primas por fluorescencia de rayos $X$.

Table I. Chemical analysis of the raw materials by $X$ ray fluorescence.

\begin{tabular}{|c|c|c|c|c|c|}
\hline & \multirow[b]{2}{*}{$\mathrm{Na}_{2} \mathrm{CO}_{3}$} & \multicolumn{4}{|c|}{ Fuentes de sílice (arenas y residuo) } \\
\hline & & Sibelco & Caobar & Microsílice & $\begin{array}{l}\text { Microsílice } \\
\quad 800^{\circ} \mathrm{C}\end{array}$ \\
\hline C & & & & 6,1 & 0,01 \\
\hline $\mathrm{Na}_{2} \mathrm{O}$ & 58,0 & 0,02 & & 0,18 & 0,19 \\
\hline $\mathrm{MgO}$ & & & & 0,32 & 0,33 \\
\hline $\mathrm{Al}_{2} \mathrm{O}_{3}$ & & 0,18 & 0,57 & 0,16 & 0,17 \\
\hline $\mathrm{SiO}_{2}$ & & 99,5 & 99,2 & 92,1 & 98,3 \\
\hline $\mathrm{K}_{2} \mathrm{O}$ & & 0,02 & 0,07 & 0,32 & 0,34 \\
\hline $\mathrm{CaO}$ & 0,03 & & 0,06 & 0,36 & 0,4 \\
\hline MnO & & & & 0,01 & 0,01 \\
\hline $\mathrm{Fe}_{2} \mathrm{O}_{3}$ & & 0,05 & 0,03 & 0,10 & 0,12 \\
\hline
\end{tabular}

obtención del silicato sódico. En la misma tabla se puede apreciar la composición de una arena de calidad (Sibelco) utilizada en la fabricación de silicato de sodio; de una arena utilizada en la industria cerámica (Caobar) y de la microsílice que se estudia como una posible fuente de sílice. Ésta última contiene un $92,1 \%$ de $\mathrm{SiO}_{2}$ y un $6,1 \%$ de carbono; las impurezas de $\mathrm{MgO}, \mathrm{Al}_{2} \mathrm{O}_{3}, \mathrm{CaO}$ y $\mathrm{Fe}_{2} \mathrm{O}_{3}$ pueden tener un efecto negativo sobre la calidad del vidrio de silicato, puesto que son formadores potenciales de compuestos insolubles. Por último, se observa un incremento en la concentración de $\mathrm{SiO}_{2}$ a 98,3 \% en la microsílice calcinada a $800^{\circ} \mathrm{C}$.

\section{RESULTADOS Y DISCUSION}

\subsection{Análisis térmico de la materia prima}

El carbonato sódico ha sido tratado térmicamente. La descomposición del carbonato comienza a llevarse a cabo en fase líquida, alrededor de los $900^{\circ} \mathrm{C}$. Esta descomposición es lenta al principio, incrementando su velocidad de descomposición por encima de $1.100{ }^{\circ} \mathrm{C}$. La pérdida de peso a $1.050^{\circ} \mathrm{C}$ y a $1.300{ }^{\circ} \mathrm{C}$ fue de $3 \%$ y $22 \%$, respectivamente. En la figura 1 se observa la curva de ATD que muestra un pico endotérmico a $863^{\circ} \mathrm{C}$ que corresponde al punto de fusión del carbonato.

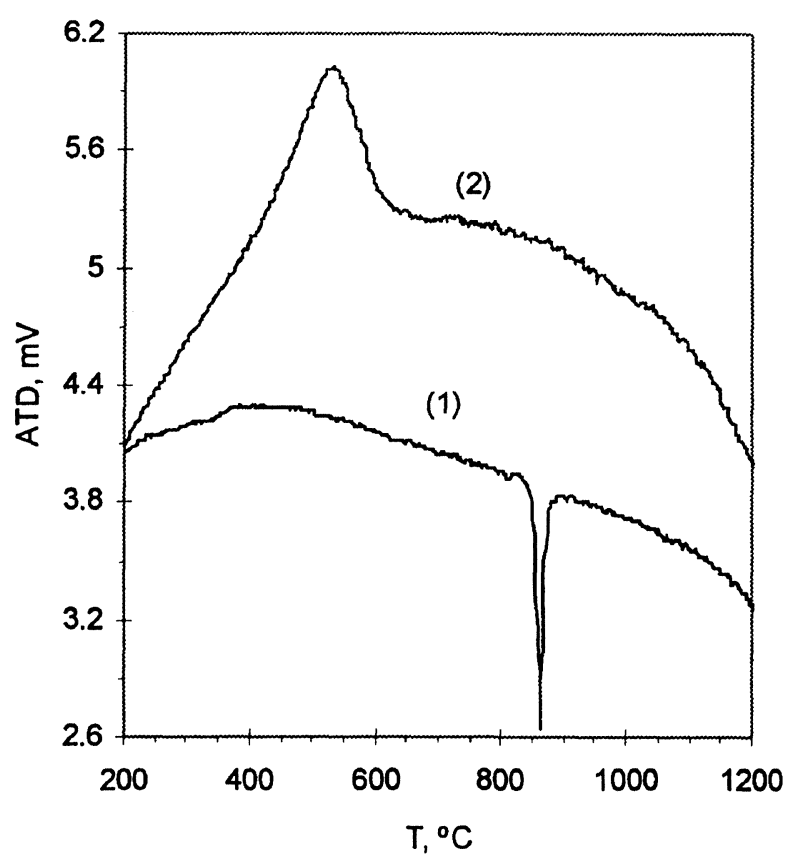

Figura 1. Resultados de ATD de $\mathrm{Na}_{2} \mathrm{CO}_{3}$ (1) y microsílice (2).

Figure 1. DTA results of $\mathrm{Na}_{2} \mathrm{CO}_{3}$ (1) and microsilica (2). 
Cuando se trata térmicamente la microsílice, se observa una pérdida de peso en el intervalo de 400-600 ${ }^{\circ} \mathrm{C}$. Esto, se debe a la eliminación de carbono, que contiene la microsílice, en forma de $\mathrm{CO}_{2}$. En la curva 2 de la figura 1 se observa un pico exotérmico que corresponde al proceso de combustión del carbono. En el intervalo de 600. $1.350^{\circ} \mathrm{C}$ se observa una pérdida de peso del $0,7 \%$, debido a la volatilización de impurezas de la microsílice.

Del análisis por DRX se ha determinado que el óxido de silicio presente en la microsílice es amorfo. La microsílice calcinada a $800{ }^{\circ} \mathrm{C}$ sigue siendo amorfa, aunque se observa un ligero cambio de fase para formar cuarzo, se observa también la presencia de óxido de hierro en forma de maguemita. A $1.350^{\circ} \mathrm{C}$, la fase predominante es la cristobalita, seguida por cuarzo, tridimita y sílice amorfa. Se sabe que las fases cristalinas de la sílice de mayor temperatura la hacen menos reactiva, por lo que estos resultados sugieren que la mejor temperatura de calcinación debe ser de $800^{\circ} \mathrm{C}$, debido a que a esta temperatura sigue predominante la sílice amorfa, con muy poca sílice cristalina, estando ésta además en forma de cuarzo, que es la más reactiva de la sílice cristalinas.

Por otro lado, en el balance de energía realizado con el programa HSC3 de Outokumpu ${ }^{[6]}$ se prevé que el uso de la microsílice sin calcinar reduce el consumo de energía debido a la reacción exotérmica por oxidación del carbono. Una de las desventajas de la microsílice sin tratar es que el carbono puede contribuir a una pérdida de sodio en fase gaseosa por reducción.

\subsection{Formación del silicato con arenas comer- ciales}

En las figuras 2 y 5 se observan las curvas de ATG y ATD para la descomposición del carbonato de sodio por interacción con la arena Caobar y Sibelco. Las proporciones en peso son tales que el modulo del producto es mayor de $3\left(\mathrm{SiO}_{2} / \mathrm{Na}_{2} \mathrm{O}\right)$. Se puede apreciar una gran diferencia en el comportamiento de descomposición del $\mathrm{Na}_{2} \mathrm{CO}_{3}$ sólo, y con la presencia de $\mathrm{SiO}_{2}$. El $\mathrm{Na}_{2} \mathrm{CO}_{3}$, mezclado con $\mathrm{SiO}_{2}$, comienza a descomponer a la temperatura de $770^{\circ} \mathrm{C}$, mientras que el $\mathrm{Na}_{2} \mathrm{CO}_{3}$ puro difícilmente descompone a esta temperatura, la velocidad de descomposición es extremadamente lenta aún después del punto de fusión $850^{\circ} \mathrm{C}$. La velocidad de descomposición del $\mathrm{Na}_{2} \mathrm{CO}_{3}$ cuando está mezclado con $\mathrm{SiO}_{2}$ se incrementa rápidamente al aumentar

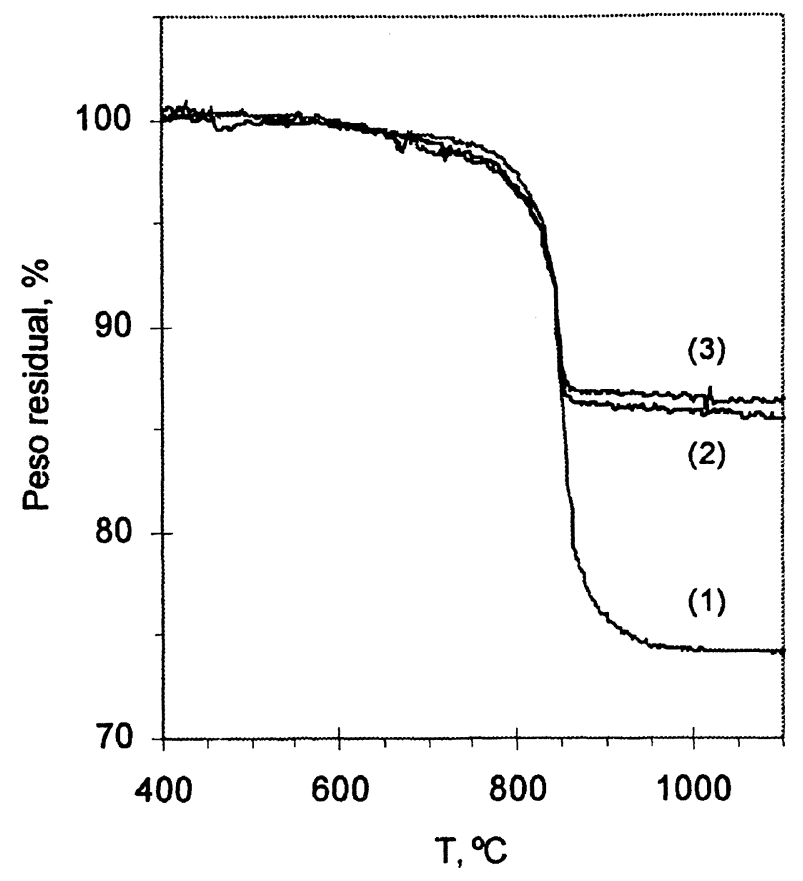

Figura 2. Resultados de ATG de las mezclas de $\mathrm{Na}_{2} \mathrm{CO}_{3}$ con sílice de Caobar (1 y 3) y de Sibelco (2).

Figure 2. GTA results with the mixture of $\mathrm{Na}_{2} \mathrm{CO}_{3}$ and Caobar (1 and 3) and Sibelco (2) silicas.

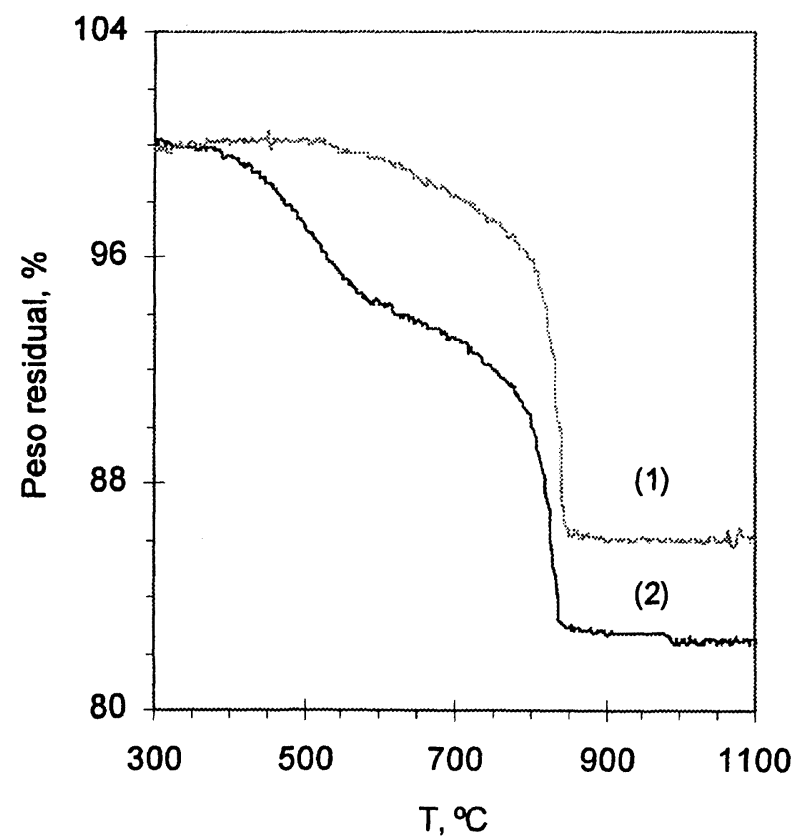

Figura 3. Pérdidas de peso para las mezclas de $\mathrm{Na}_{2} \mathrm{CO}_{3}$ con microsílice calcinada (1) y sin calcinar (2).

Figure 3. Mass loss for the mixture of $\mathrm{Na}_{2} \mathrm{CO}_{3}$ and calcinated microsilica (1) and without (2).

la temperatura. La velocidad de la pérdida de peso alcanza el valor máximo cuando la temperatura se aproxima al punto de fusión del $\mathrm{Na}_{2} \mathrm{CO}_{3}$ (Fig. 4) 


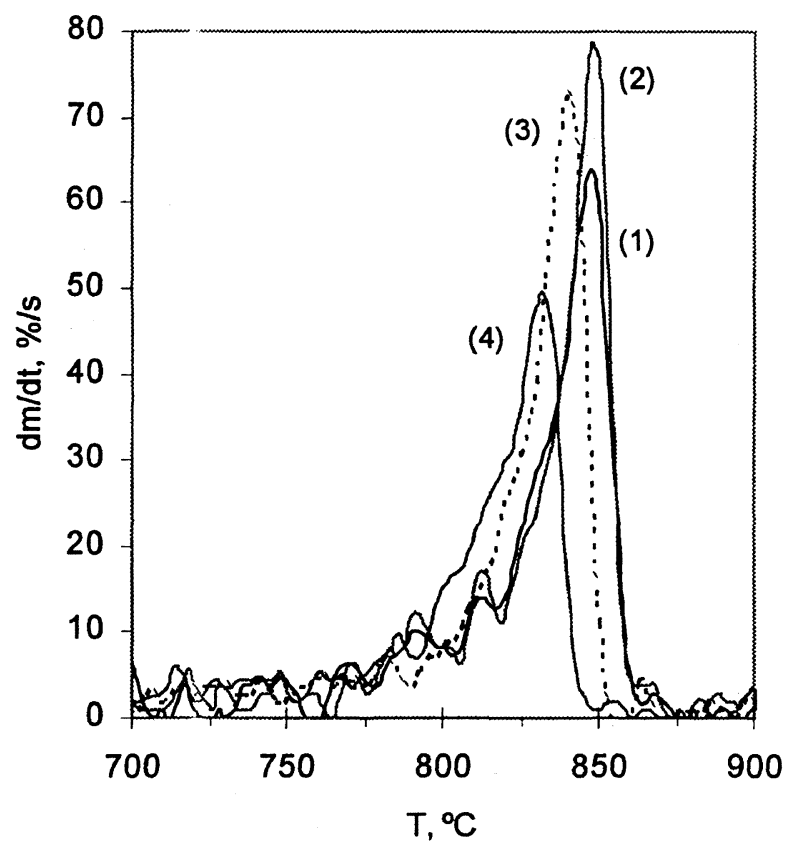

Figura 4. Cambios en la velocidad de pérdida de peso debido a la descomposición de $\mathrm{Na}_{2} \mathrm{CO}_{3}$ con $\mathrm{SiO}_{2}$ : (1) Caobar, (2) Sibelco, (3) microsílice calcinada, (4) microsílice.

Figure 4. Changes in the rate of mass loss during $\mathrm{Na}_{2} \mathrm{CO}_{3}$ decomposition with $\mathrm{SiO}_{2}$ : (1) Caobar, (2) Sibelco, (3) calcinated microsilica and (4) microsilica.

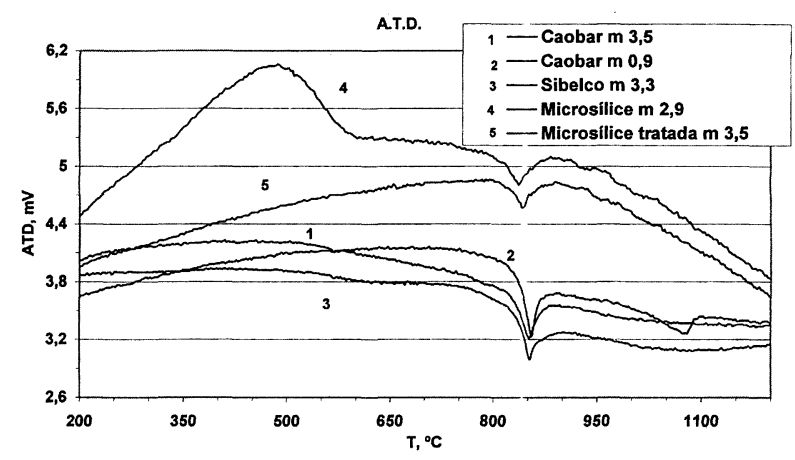

Figura 5. Resultados de ATD de las mezclas de $\mathrm{Na}_{2} \mathrm{CO}_{3}$ con diferentes fuentes de sílice.

Figure 5. DTA results with the mixture of $\mathrm{Na}_{2} \mathrm{CO}_{3}$ and several $\mathrm{SiO}_{2}$ sources.

que corresponde al pico endotérmico de las curvas 1 y 3 (Fig. 5).

Las figuras. 2 y 4 también muestran que la descomposición del $\mathrm{Na}_{2} \mathrm{CO}_{3}$ con $\mathrm{SiO}_{2}$ es casi completa inmediatamente después que la temperatura haya alcanzado el punto de fusión del carbonato.

En la figura 2 (curvas 2 y 3 ) se observa que la descomposición del carbonato comienza a producirse alrededor de los $800^{\circ} \mathrm{C}$. Esta temperatura corresponde al punto eutéctico $\left(789^{\circ} \mathrm{C}\right)$ del sistema
$\mathrm{Na}_{2} \mathrm{O}-\mathrm{SiO}_{2}\left(73,4 \% \mathrm{SiO}_{2}\right)^{[7]}$. Esta coincidencia sugiere que la reacción de descomposición principal del $\mathrm{Na}_{2} \mathrm{CO}_{3}$ tiene lugar cuando se forma una fase líquida de $\left(\mathrm{Na}_{2} \mathrm{O}+\mathrm{SiO}_{2}\right)$ en la interfase $\mathrm{Na}_{2} \mathrm{CO}_{3}(\mathrm{~s}) / \mathrm{SiO}_{2}(\mathrm{~s})$. Una vez que la fase líquida se ha formado, se producen, simultáneamente, las siguientes etapas que son propuestas por Kim y Lee ${ }^{[8]}$ : (i) disolución de $\mathrm{SiO}_{2}$ en la fase líquida en la interfase $\mathrm{SiO}_{2} /$ líquido; (ii) formación de metasilicato de sodio $\left(\mathrm{Na}_{2} \mathrm{SiO}_{3}(\mathrm{~s})\right)$ debido a la emisión de $\mathrm{CO}_{2}$; (iii) disolución de una porción de $\mathrm{Na}_{2} \mathrm{SiO}_{3}$ (s) en la fase líquida. La velocidad de este proceso mejora con la temperatura hasta que no quede, en nuestro caso, $\mathrm{Na}_{2} \mathrm{CO}_{3}$, como se observa en las figuras 2 y 4.

En el caso de que la relación molar $\mathrm{SiO}_{2} / \mathrm{Na}_{2} \mathrm{O}$ sea próxima a 1 (curva 1 de la figura 2), el producto de descomposición será sólo $\mathrm{Na}_{2} \mathrm{SiO}_{3}$. Una evidencia de la formación de $\mathrm{Na}_{2} \mathrm{SiO}_{3}$ se obtiene en el análisis térmico diferencial para la muestra de relación en peso $\mathrm{SiO}_{2} / \mathrm{Na}_{2} \mathrm{O}$ de 0,9 como se muestra en la curva 2 de la figura 5 . El pico endotérmico a $1082{ }^{\circ} \mathrm{C}$ corresponde al punto de fusión del $\mathrm{Na}_{2} \mathrm{SiO}_{3}$. Esto sugiere que el $\mathrm{Na}_{2} \mathrm{SiO}_{3}$ se forma en el curso de la descomposición del $\mathrm{Na}_{2} \mathrm{CO}_{3}$ en presencia de $\mathrm{SiO}_{2}$ y funde cuando la temperatura excede su punto de fusión. En nuestro caso, con una relación en peso $\mathrm{SiO}_{2} / \mathrm{Na}_{2} \mathrm{O}$ mayor de 3, todo el $\mathrm{Na}_{2} \mathrm{CO}_{3}$ se descompone y el $\mathrm{Na}_{2} \mathrm{SiO}_{3}$ formado se disolverá en la fase líquida. $\mathrm{La} \mathrm{SiO}_{2}(\mathrm{~s})$ residual será digerida en la fase líquida conforme se incremente la temperatura, elevando el módulo del silicato sódico.

\subsection{Formación de silicato a partir de un resi- duo de sílice}

Se pretende utilizar la microsílice como una materia prima alternativa para la fabricación de vidrio de silicato. Los resultados del análisis térmico al utilizar la microsílice se observan en las figuras 3 y 5. Se analizaron dos casos: microsílice tal como se obtiene en la fabricación de Silicio metal y microsílice calcinada previamente a $800^{\circ} \mathrm{C}$.

En las figuras 2 y 3 , se observan que las curvas de pérdida de peso por descomposición del $\mathrm{Na}_{2} \mathrm{CO}_{3}$ son parecidas que cuando se utiliza arena comercial de Sibelco y Caobar. Cabe resaltar que al usar microsílice se observa una pérdida de peso en el intervalo de temperatura $400-600{ }^{\circ} \mathrm{C}$, debido a la combustión del carbono inherente a la microsílice, cuyo pico exotérmico se observa aproximadamente a $500{ }^{\circ} \mathrm{C}$. Esta reacción de 
combustión antecede a la de descomposición del $\mathrm{Na}_{2} \mathrm{CO}_{3}$.

Una vez que ha culminado la reacción de combustión, la curva presenta un comportamiento igual a la de las demás muestras. Se puede observar que la descomposición del $\mathrm{Na}_{2} \mathrm{CO}_{3}$ comienza y se completa a temperaturas ligeramente inferiores que cuando se usa arena comercial. Este efecto se observa mejor al utilizar microsílice sin tratamiento previo (ver Fig. 4). Esta mejora se debe, principalmente, a la granulometría muy fina de la microsílice, lo que acelera el proceso de descomposición del carbonato, anticipando la formación de una fase líquida que, además, se ve favorecida por la formación de silicatos de algunas impurezas como el hierro, de bajo punto de fusión.

Bauer ${ }^{[9]}$ ha determinado que si se tiene una atmósfera reductora dentro de un horno de vidrio se incrementará la formación de vapor de sodio, $\mathrm{Na}(\mathrm{g})$. El estudio termodinámico realizado con el programa HSC3 prevé que al utilizar la microsílice, que contiene carbono y algunos elementos minoritarios en forma reducida, puede contribuir a generar más $\mathrm{Na}(\mathrm{g})$ en atmósfera oxidante que el $\mathrm{Na}(\mathrm{g})$ generado al usar arena comercial. Este efecto puede producirse, principalmente, en la zona inferior del lecho de materia prima donde se puede formar un ambiente reductor, debido al carbono presente en la microsílice, incrementando la formación de $\mathrm{Na}(\mathrm{g})$. Este efecto comienza a producirse a partir de los $1.350^{\circ} \mathrm{C}$.

Los vidrios obtenidos tienen una coloración oscura provocada por impurezas de manganeso y hierro contenidas en la microsílice. Sin embargo, esto no afecta la transparencia de la disolución. Los rendimientos de disolución fueron próximos al $80 \%$. Estos resultados no reproducen la influencia negativa al usar la microsílice, debido a que los compuestos insolubles formados provienen, generalmente, del material refractario utilizado.

\section{CONCLUSIONES}

Mediante el análisis térmico se ha investigado la descomposición del carbonato de sodio al inter- accionar con la microsílice, residuo de la fabricación del silicio metal, formando silicato de sodio.

Las conclusiones se resumen en lo siguiente:

- La descomposición del carbonato sódico es fuertemente acelerada por la interacción con una fuente de sílice, en nuestro caso, por la microsílice. Se establece que la mayor velocidad de descomposición ocurre en el intervalo de temperatura entre $800-860^{\circ} \mathrm{C}$.

- La velocidad de descomposición es independiente de la relación $\mathrm{SiO}_{2} / \mathrm{Na}_{2} \mathrm{O}$, y ocurre hasta que la descomposición del carbonato se haya agotado.

- La utilización de la microsílice adelanta ligeramente el intervalo de la temperatura de descomposición del carbonato sódico, además de reducir el consumo energético para la preparación del silicato sódico por la energía producida por oxidación del carbono inherente en la microsílice. Es viable la utilización de la microsílice como fuente de $\mathrm{SiO}_{2}$ para el proceso de fabricación de vidrio soluble.

\section{REFERENCIAS}

[1] B.M. Coope, Ind. Miner. (1989) 43-55.

[2] KiRK-OTHMER, Encyclopedia of chemical technology, Vol 22, John Wiley \& Sons (1997).

[3] G. Pacheco, C. Piña, J.L. España y J. Baez, Bol. Soc. Esp. Ceram. Vidr. 31 (1992) 131-133.

[4] J.N. Ayala, Tesis Doctoral, UPM, Madrid, 1991.

[5] R. Quintana, L. Perdomo, A. Cruz, L. Gómez, L.L. García, A. Cerpa y A. Cores: Rev. Metal. Madrid 40 (2004) 294-303.

[6] A. RoINE, In Outokumpu HSC chemistry for windows, versión 3,0, Finland (1997).

[7] J.M. Fernandez, El Vidrio, Consejo Superior de Investigaciones Científicas, Madrid, 1991.

[8] J.W. KIM, Y.D. LEE y H.G. LeE, ISIJ Int. 41 (2001) 116-123.

[9] R. BAuer, A.M. LANKHORST y J.A.C. VAN, Int. Glass J. 99 (1999) 28-32. 